\title{
A difference between auditory and visual apparent movement
}

\author{
PAUL A. KOLERS \\ University of Toronto, Toronto, Ontario, Canada M5S 1A1
}

\begin{abstract}
Disparate shapes presented visually undergo continuous transformation in apparent motion, whereas disparate colors undergo abrupt transformation. Analogous functions were sought in the auditory domain between pitches, volumes, and timbres. No continuous transformations were experienced; all changes were abrupt.
\end{abstract}

The mechanism mediating apparent motion must be fundamental (basic or primitive) to perception, for illusory motion can be found in all the sense departments: vision, audition, touch, and so on. In fact, it was soon after Wertheimer (1912/1961) published his fundamental paper that Burt (1917a, 1917b) challenged the proposed theory by demonstrating apparent motion not only between the ears, which project to both cerebral hemispheres, but also between the hands, which do not. Von Békésy (1967), among others, pursued the study into other modalities.

Despite the ubiquity of the phenomenon, its form is not always the same even within a modality. When outline drawings are presented to the visual system at the proper spatial and temporal separations, they seem to change shape as they move, and the change of shape is usually smooth and continuous (Kolers, 1972). If the two presentations differ in color, however, the result is quite different. While two like-colored squares are seen in smooth, continuous movement, a red square alternated with a green square seems to undergo an abrupt shift from red to green; the same abruptness is found with all other disparities of color and brightness (Kolers \& von Grunau, 1976). Does the distinction between abrupt and continuous transformations characterize a more persuasive principle? The present note describes an analogous test for audition.

What is the auditory analog of visual shape and of visual color? In the classic distinction between modality and attribute traced to Helmholtz, the pitch of a tone is the analog of the color of a visual stimulus (their quality), and the volume or fullness of a tone is the analog of the shape or figure (their extension) (Boring, 1933).

This work was supported by Grant A7655 from the National Research Council and by Grant W760174 from the Canada Council. The work was carried out in the Bio-Communications Laboratory, School of Engineering, Tel Aviv University, with the kind cooperation of the Director, Dr. Julius Goldstein, and with the assistance of Dr. Allen Gerson. I am grateful too to Proffessor Giovanni Vicario of the University of Padua for many useful discussions of the subject. Author's address: Department of Psychology, University of Toronto, Toronto, Ontario, Canada M5S 1A1.
In the present tests, two different pitches were presented, one to each ear, to learn whether their apparent movement was of a single, continuously changing pitch or of the abrupt replacement of one pitch by another. Two tones of different amplitude were presented, one to each ear, similarly. In addition, two tones of the same apparent pitch but different timbre (another definition of tone color) were presented, one to each ear.

A Varian 620-L-100 computer generated the tones, controlled their timing, and collected and processed the data. An observer seated in an IAC acoustic room heard the presentations through a pair of TDH 39 stereophonic earphones. The range of pitches was 600 to $1,400 \mathrm{~Hz}$, between 60 and $100 \mathrm{~dB} \mathrm{SPL}$. The tones were sinusoids with square onsets and offsets. Different timbres were produced by a composition of 400,600 , and $800 \mathrm{~Hz}$ in one ear and of $800,1,000$, and $1,200 \mathrm{~Hz}$ in the other, the perceptual effect being two tones of $200 \mathrm{~Hz}$ each but of different color.

Two observers were first tested for their ability to hear auditory apparent movement. The two tones were square-wave onsets of $1,000 \mathrm{~Hz}$ at $80 \mathrm{~dB} \mathrm{SPL}$ and 50 -msec duration. The interval separating the two ranged from 0 to $250 \mathrm{msec}$. The curve of Figure 1 shows the likelihood of hearing a single sound move "through the head." The curve is similar in shape to that

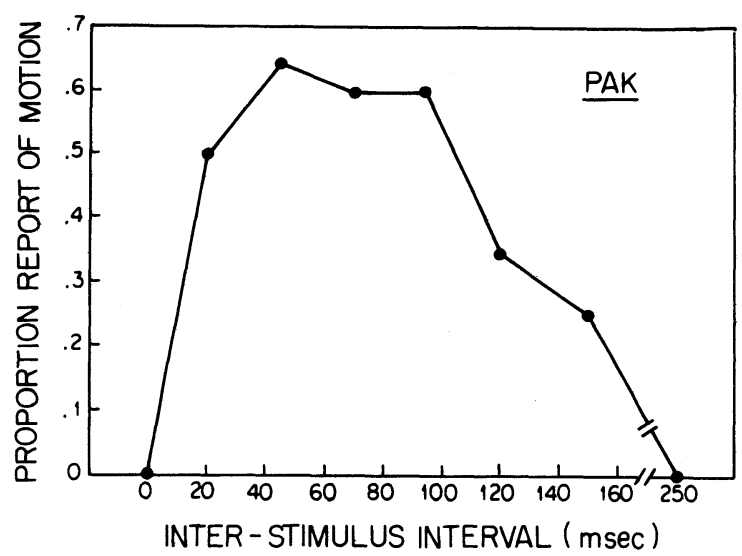

Figure 1. Likelihood of reporting auditory apparent motion. 
found with visual apparent motion (Kolers, 1972). The similarity extends to the fact (not shown in the figure) that the likelihood of motion perception increased with an increase in the intensity of the tones, which were almost never heard when the intensity was $60 \mathrm{~dB}$ SPL but were heard with increasing frequency as intensity was increased to $100 \mathrm{~dB}$ SPL.

The major interest was in the nature of the transition when the pitches, their volume, or their timbre differed. For this purpose, a neon glow lamp flashed for $5 \mathrm{msec}$ at varied moments during the interstimulus interval, with the duration of the tones and the interstimulus interval kept constant at about $50 \mathrm{msec}$ each. The lamp appeared immediately after the offset of the first tone, immediately before the onset of the second, or at varied points during the separation. The observers described whether when the light had appeared, the tone had the pitch of the onset, of the terminus, or of some other tone; similar descriptions were required for volume and for timbre.

For both subjects tested, the apparently moving tone seemed to pass either through the head or over or around the head. (Earphones rather than loud speakers were used to preclude the influence of reverberation and other aspects of room acoustics on the perceptual processing.) When the tones differed in frequency, a tone was heard at one pitch as it seemed to leave one location and at the other pitch when it arrived at the terminus of movement, and similarly for volume and timbre. The transition between the pitches, volumes, or timbres was always abrupt, however. The perceptual experience was of a tone of a certain pitch, timbre, or volume shifting abruptly during the movement to the other pitch, timbre, or volume, with no grading between the two tones. Localizing the point of transition by reference to the briefly presented glow lamp was very difficult to accomplish; that difficulty notwithstanding, the perceptual fact of abruptness was obvious.
The finding, then, is that in the visual domain there is a functional difference in the processing of shape and color but that analogous functions are not found among the tested dimensions in hearing. One might question the adequacy of the classic equation of visual color with auditory pitch and visual shape with auditory volume (Miller \& Johnson-Laird, 1976); perhaps pattern or sequence of tones is more nearly the analog of shape (Goodman, 1951), or even their phase relations. Perhaps, too, shapes felt on the skin may be found to change in a continuous way. It would be curious indeed if continuity of perceptual transformation were restricted to two-dimensional drawings presented visually and all other disparate stimuli were resolved by abrupt transformation.

\section{REFERENCES}

Boring, E. G. The physical dimensions of consciousness. New York: Century, 1933.

BURT, H. E. Auditory illusions of movement-a preliminary study. Journal of Experimental Psychology, 1917, 2, 63-75. (a)

BURT, H. E. Tactual illusions of movement. Journal of Experimental Psychology, 1917, 2, 371-385. (b)

Goodman, N. The structure of appearance. Cambridge, Mass: Harvard University Press, 1951.

Kolers, P. A. Aspects of motion perception. Oxford: Pergamon Press, 1972.

Kolers, P. A., \& von GrunaU, M. Shape and color in apparent motion. Vision Research, 1976, 16, 329-335.

Miller, G. A., \& Johnson-Lamd, P. N. Language and perception. Cambridge, Mass: Harvard University Press, 1976.

von Békésy, G. Sensory inhibition. Princeton, N.J: Princeton University Press, 1967.

Wertheimer, M. Experimentelle Studien über das Sehen von Bewegung. Zeitschrift für Psychologie, 1912, 61, 161-265. Trans. in part in T. Shipley (Ed.), Classics in psychology. New York: Philosophical Library, 1961.

(Received for publication January 31, 1979.) 